\title{
THE INFLUENCE OF PROTEIN INTAKE ON THE UREA CLEARANCE IN NORMAL MAN
}

\author{
By WILLIAM GOLDRING, LOUIS RAZINSKY,1 MONROE GREENBLATT, ${ }^{1}$ \\ AND SIDNEY COHEN 1
}

\section{(From the Department of Medicine, University and Bellevue Hospital Medical College, New York University and the Third (New York University) Medical Division Bellerue Hospital. New York)}

(Received for publication May 22, 1934)

Cope (1) has reported that a change in protein content of the diet from 75 grams to 40 grams per day is accompanied by a lowering of the urea clearance in nephritics, who had approximately normal urea clearances during the control period. No doubt Cope's investigations, like our own, were stimulated in part by the observations of Jolliffe and Smith (2) that in dogs the urea clearance may be raised by over 100 per cent by change from a low protein to a high protein diet. Since the effect of diet in Cope's experiments appears to be much less marked than is the case in the dog, it was felt that the problem merited further investigation in normal man to avoid the possibility of overlooking a functional response that might be depressed in a structurally diseased kidney. Consequently we have repeated these experiments upon subjects without renal, cardiac or vascular diseases and with no recent febrile reaction (3). As a further modification of Cope's experiments the protein intake has been varied from 9 grams to 280 grams per day, and in most instances the rate of urine excretion has been above the augmentation limit ( 2 cc. per minute).

The subjects were volunteers selected from the wards of the Third (New York University) Medical Division of Bellevue Hospital. Urea clearances were performed in the morning without breakfast, with the patient recumbent in bed. During the previous night and up to two hours before the discard preceding the first urine collection period, the subject was given 3,000 to $4,000 \mathrm{cc}$. of water to insure high rates of urine flow. An effort was made to avoid collection of urine in the ascending phase or at the peak of diuresis. All discards and urine specimens were collected by catheterization to insure complete emptying of the bladder. Blood was drawn from the median basilic vein at the beginning, middle and end of each experiment. When the three blood urea values, as separately determined, checked within three per cent, they were averaged, and when they did not, values interpolated to the middle of each period were used. Urea in plasma and in urine were determined in duplicate by the gasometric

${ }^{1}$ Graduate Students in the Department of Medicine. 
UREA CLEARANCE

TABLE I

Data of urea clearances

\begin{tabular}{|c|c|c|c|c|c|}
\hline & $\begin{array}{c}\text { Daily } \\
\text { protein } \\
\text { intake }\end{array}$ & Date & $\begin{array}{c}\mathrm{V} \\
\text { Urine } \\
\text { volume }\end{array}$ & $\underset{\substack{\text { Plasma } \\
\text { urea }}}{\mathbf{P}}$ & $\frac{U V}{\text { clearance }}$ \\
\hline \multirow{6}{*}{$\begin{array}{l}\text { M. O., age } 40, \text { surface } \\
\text { area } 1.7 \text { sq. m. }\end{array}$} & grams & & $\begin{array}{l}c c . \text { per } \\
\text { minule }\end{array}$ & $\underset{\text { per cent }}{\operatorname{mgm.}}$ & $\begin{array}{c}c c . \text { per sq. m. } \\
\text { per minule }\end{array}$ \\
\hline & 100 & $\begin{array}{l}\text { November } 7,1933 \\
\text { November } 9,1933\end{array}$ & $\begin{array}{r}3.59 \\
5.00 \\
8.31 \\
3.56 \\
17.29 \\
16.50 \\
15.20 \\
11.35\end{array}$ & $\begin{array}{l}18.9 \\
19.0 \\
19.0 \\
18.99 \\
21.15 \\
21.39 \\
21.05 \\
20.07\end{array}$ & \begin{tabular}{|l|}
38.9 \\
57.0 \\
58.0 \\
44.1 \\
47.8 \\
40.4 \\
49.1 \\
44.5
\end{tabular} \\
\hline & \multicolumn{2}{|c|}{ Average } & & & 47.5 \\
\hline & 9 & $\begin{array}{l}\text { November 15, } 1933 \\
\text { November } 17,1933\end{array}$ & $\begin{array}{l}12.07 \\
13.68 \\
13.07 \\
12.50 \\
12.00 \\
11.46 \\
11.16 \\
11.80\end{array}$ & $\begin{array}{l}5.93 \\
6.22 \\
6.15 \\
6.10 \\
6.95 \\
6.52 \\
6.51 \\
6.50\end{array}$ & \begin{tabular}{|l|}
34.6 \\
38.9 \\
39.1 \\
36.6 \\
36.4 \\
37.4 \\
32.8 \\
35.3
\end{tabular} \\
\hline & \multicolumn{2}{|c|}{ Average } & & & 36.3 \\
\hline & 280 & $\begin{array}{l}\text { November 23, } 1933 \\
\text { November } 25,1933 \\
\text { November 28, } 1933\end{array}$ & $\begin{array}{l}19.00 \\
15.70 \\
15.75 \\
14.80 \\
15.70 \\
16.00 \\
13.40 \\
13.27 \\
18.25 \\
17.15 \\
14.90 \\
14.72\end{array}$ & $\begin{array}{l}37.89 \\
37.69 \\
37.05 \\
37.55 \\
34.10 \\
33.61 \\
33.30 \\
32.96 \\
41.30 \\
39.80 \\
38.70 \\
37.90\end{array}$ & $\begin{array}{l}53.0 \\
34.8 \\
47.2 \\
50.0 \\
44.8 \\
51.9 \\
43.2 \\
46.1 \\
48.2 \\
49.5 \\
43.6 \\
45.3\end{array}$ \\
\hline \multirow{3}{*}{$\begin{array}{l}\text { P. M., age } 48 \text { years, } \\
\text { surface area } 1.84 \\
\text { sq. } m \text {. }\end{array}$} & \multicolumn{2}{|c|}{ Average } & & & 46.4 \\
\hline & 100 & $\begin{array}{l}\text { December } 6,1933 \\
\text { December } 8,1933\end{array}$ & $\begin{array}{l}4.28 \\
4.28 \\
5.48 \\
7.71 \\
4.52 \\
5.12 \\
3.82 \\
2.60\end{array}$ & $\begin{array}{l}23.90 \\
23.50 \\
23.38 \\
23.28 \\
25.86 \\
25.80 \\
.25 .65 \\
25.50\end{array}$ & $\begin{array}{l}54.6 \\
56.8 \\
55.1 \\
61.5 \\
57.4 \\
59.4 \\
50.1 \\
42.9\end{array}$ \\
\hline & \multicolumn{2}{|c|}{ Average } & & & 54.8 \\
\hline
\end{tabular}


method of Van Slyke (4). Heparin was used as an anticoagulant. All patients were attended by a special nurse and the diets were prepared by a trained dietician attached to the teaching unit of New York University Medical Service.

The control urea clearances were determined while patients were re- . ceiving the usual ward diet containing an average of 100 grams of protein

\begin{tabular}{|c|c|c|c|c|c|}
\hline & $\mid \begin{array}{c}\text { Daily } \\
\text { protein } \\
\text { intake }\end{array}$ & Date & $\begin{array}{c}\mathrm{V} \\
\text { Urine } \\
\text { volume }\end{array}$ & $\underset{\text { urea }}{\stackrel{P}{\text { Plasma }}}$ & $\frac{U V}{\text { clearance }}$ \\
\hline \multirow[t]{4}{*}{ P. M. (continued) } & $\begin{array}{c}\text { grams } \\
9\end{array}$ & $\begin{array}{l}\text { December } 13,1933 \\
\text { December } 15,1933\end{array}$ & $\begin{array}{c}c c . \text { per } \\
\text { minute } \\
3.73 \\
4.64 \\
4.23 \\
3.07 \\
4.86 \\
3.20 \\
3.10 \\
3.30\end{array}$ & 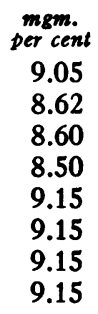 & $\begin{array}{c}c c . \text { per sq. m. } \\
\text { per minute } \\
43.6 \\
54.2 \\
47.7 \\
38.2 \\
48.3 \\
46.9 \\
44.3 \\
42.5\end{array}$ \\
\hline & \multicolumn{2}{|c|}{ Average } & & & 45.6 \\
\hline & 280 & $\begin{array}{l}\text { December 20, } 1933 \\
\text { December 22, } 1933\end{array}$ & $\begin{array}{l}2.75 \\
3.70 \\
4.60 \\
4.63 \\
6.04 \\
4.80 \\
5.08 \\
3.43\end{array}$ & $\begin{array}{l}41.37 \\
40.84 \\
40.32 \\
39.83 \\
38.60 \\
38.94 \\
38.45 \\
38.05\end{array}$ & $\begin{array}{l}48.0 \\
55.3 \\
56.3 \\
56.1 \\
59.6 \\
62.3 \\
57.4 \\
55.2\end{array}$ \\
\hline & \multicolumn{2}{|c|}{ Average } & & & 56.0 \\
\hline \multirow[t]{6}{*}{$\begin{array}{l}\text { J. G., } \dagger \text { age } 37 \text { years, } \\
\text { surface area } 1.66 \\
\text { sq. } \mathrm{m} \text {. }\end{array}$} & 9 & December 16, 1933 & $\begin{array}{l}6.41 \\
4.23 \\
3.37 \\
3.50\end{array}$ & $\begin{array}{l}8.42 \\
8.42 \\
8.42 \\
8.42\end{array}$ & $\begin{array}{l}31.7 \\
32.5 \\
28.6 \\
30.4\end{array}$ \\
\hline & \multicolumn{2}{|c|}{ Average } & & & 30.8 \\
\hline & 280 & December 22, 1933 & $\begin{array}{r}11.35 \\
10.45 \\
7.36 \\
3.06\end{array}$ & $\begin{array}{l}47.35 \\
46.70 \\
46.30 \\
45.90\end{array}$ & \begin{tabular}{|l|}
33.2 \\
40.3 \\
38.6 \\
30.1
\end{tabular} \\
\hline & \multicolumn{2}{|c|}{ Average } & & & 35.5 \\
\hline & 100 & December 29, 1933 & $\begin{array}{r}12.65 \\
12.35 \\
10.90 \\
9.18\end{array}$ & $\begin{array}{l}24.05 \\
24.30 \\
23.75 \\
23.35\end{array}$ & $\begin{array}{l}34.2 \\
38.5 \\
36.7 \\
34.3\end{array}$ \\
\hline & \multicolumn{2}{|c|}{ Average } & & & 35.9 \\
\hline
\end{tabular}


per day. Eight clearances were determined on two different days. Following the last control observation the patient was given a diet containing 9 grams of protein per day. On the sixth day four clearances were determined, and on the eighth day, four more. The same procedure was fol-

TABLE I (continued)

\begin{tabular}{|c|c|c|c|c|c|c|}
\hline & $\begin{array}{c}\text { Daily } \\
\text { protein } \\
\text { intake }\end{array}$ & \multicolumn{2}{|c|}{ Date } & $\begin{array}{c}\mathrm{V} \\
\text { Urine } \\
\text { volume }\end{array}$ & $\underset{\substack{\text { Plasma } \\
\text { urea }}}{\mathbf{P}}$ & $\frac{\mathrm{UV}}{\mathbf{P ( S A )}}$ \\
\hline \multirow[t]{6}{*}{$\begin{array}{l}\text { M. C., age } 40 \text { years, } \\
\text { surface area } 2.08 \\
\text { sq. m. }\end{array}$} & $\begin{array}{c}\text { grams } \\
100\end{array}$ & $\begin{array}{l}\text { January } \\
\text { February }\end{array}$ & $\begin{array}{l}1,1934 \\
2,1934\end{array}$ & $\begin{array}{r}c c . \text { per } \\
\text { minute } \\
4.67 \\
5.50 \\
5.13 \\
5.20 \\
23.05 \\
20.95 \\
21.20 \\
20.60\end{array}$ & 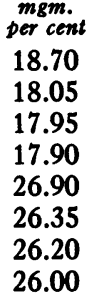 & $\begin{array}{l}\text { cc. per sq. m. } \\
\text { per minute } \\
28.5 \\
44.1 \\
36.4 \\
35.0 \\
42.2 \\
39.6 \\
41.4 \\
42.1\end{array}$ \\
\hline & \multicolumn{3}{|c|}{ Average } & & & 38.6 \\
\hline & 9 & $\begin{array}{l}\text { February } \\
\text { February }\end{array}$ & $\begin{array}{l}7,1934 \\
9,1934\end{array}$ & $\begin{array}{l}6.77 \\
6.10 \\
6.54 \\
4.80 \\
5.63 \\
4.95 \\
4.08 \\
4.00\end{array}$ & $\begin{array}{l}8.54 \\
8.83 \\
9.01 \\
9.26 \\
6.57 \\
6.57 \\
6.57 \\
6.57\end{array}$ & $\begin{array}{l}24.5 \\
24.8 \\
31.5 \\
23.6 \\
25.4 \\
23.6 \\
21.1 \\
22.0\end{array}$ \\
\hline & \multicolumn{3}{|c|}{ Average } & & & 24.6 \\
\hline & 280 & $\begin{array}{l}\text { February } \\
\text { February } \\
\text { February } \\
\text { February } \\
\text { March }\end{array}$ & $\begin{array}{l}14,1934 \\
16,1934 \\
21,1934 \\
28,1934\end{array}$ & $\begin{array}{l}4.52 \\
6.73 \\
2.85 \\
2.64 \\
5.80 \\
2.45 \\
2.69 \\
5.35 \\
6.57 \\
3.17 \\
3.15 \\
2.62 \\
2.73 \\
4.77 \\
7.73 \\
6.87 \\
1.73 \\
1.55 \\
1.80 \\
1.74\end{array}$ & $\begin{array}{l}38.40 \\
38.40 \\
38.40 \\
38.40 \\
41.18 \\
39.95 \\
39.78 \\
39.43 \\
39.50 \\
39.50 \\
39.50 \\
39.50 \\
36.61 \\
35.90 \\
35.63 \\
35.23 \\
40.40 \\
40.40 \\
40.40 \\
40.40\end{array}$ & $\begin{array}{l}44.8 \\
45.4 \\
30.5 \\
34.9 \\
29.7 \\
25.6 \\
33.1 \\
46.7 \\
33.1 \\
34.3 \\
33.1 \\
32.4 \\
31.9 \\
43.9 \\
45.4 \\
36.2 \\
21.9^{*} \\
25.7^{*} \\
27.4^{*} \\
27.1^{*}\end{array}$ \\
\hline & \multicolumn{3}{|c|}{ Average } & & & 36.3 \\
\hline
\end{tabular}


lowed on the high protein diet which contained 280 grams of protein. The protein in this diet was composed principally of meat, cheese and milk products, meat constituting about one-third. On one subject (M. C.) clearances were determined on a high protein diet over a period of twentyfive days. A total of 124 urea clearances were determined on five subjects, and with five exceptions the urine flow was above $2 \mathrm{cc}$. per minute. The results of these observations are given in Table I.

The greatest reduction in clearance observed on the low protein diet was 36 per cent below the control, and the average reduction was 23 per cent. We have no doubt that this change is significant and reflects an

TABLE I (continued)

\begin{tabular}{|c|c|c|c|c|c|c|}
\hline & $\begin{array}{c}\text { Daily } \\
\text { protein } \\
\text { intake }\end{array}$ & \multicolumn{2}{|c|}{ Date } & $\begin{array}{c}\text { V } \\
\text { Urine } \\
\text { volume }\end{array}$ & $\underset{\text { Plasma }}{\mathbf{P}}$ & $\frac{\mathrm{UV}}{\begin{array}{c}\mathrm{P}(\mathrm{SA}) \\
\text { clearance }\end{array}}$ \\
\hline \multirow[t]{6}{*}{$\begin{array}{l}\text { J. J., age } 45 \text { years, } \\
\text { surface area } 1.68 \\
\text { sq. m. }\end{array}$} & 100 & $\begin{array}{l}\text { Februar } \\
\text { March }\end{array}$ & $\begin{array}{r}26,1934 \\
2,1934\end{array}$ & $\begin{array}{l}5.42 \\
2.93 \\
2.30 \\
2.64 \\
3.27 \\
2.85 \\
2.086 \\
1.76\end{array}$ & $\begin{array}{l}19.70 \\
19.50 \\
19.30 \\
19.20 \\
17.70 \\
17.70 \\
17.70 \\
17.70\end{array}$ & $\begin{array}{l}35.1 \\
38.6 \\
36.1 \\
43.3 \\
34.8 \\
38.0 \\
30.8 \\
22.4^{*}\end{array}$ \\
\hline & \multicolumn{3}{|c|}{ Average } & & & 35.2 \\
\hline & 9 & $\begin{array}{l}\text { March } \\
\text { March }\end{array}$ & $\begin{array}{l}7,1934 \\
9,1934\end{array}$ & $\begin{array}{r}11.00 \\
8.14 \\
5.91 \\
3.68 \\
12.10 \\
10.42 \\
10.10 \\
8.00\end{array}$ & $\begin{array}{l}5.76 \\
5.10 \\
5.18 \\
5.28 \\
7.47 \\
7.43 \\
6.91 \\
6.47\end{array}$ & $\begin{array}{l}28.9 \\
31.3 \\
29.0 \\
28.9 \\
26.2 \\
24.1 \\
26.0 \\
28.1\end{array}$ \\
\hline & \multicolumn{3}{|c|}{ Average } & & & 27.8 \\
\hline & 280 & $\begin{array}{l}\text { March } \\
\text { March }\end{array}$ & $\begin{array}{l}14,1934 \\
16,1934\end{array}$ & $\begin{array}{l}2.75 \\
3.23 \\
2.36 \\
1.92 \\
2.53 \\
2.45 \\
2.42 \\
2.53\end{array}$ & $\begin{array}{l}44.40 \\
43.10 \\
42.10 \\
40.80 \\
46.42 \\
45.98 \\
45.08 \\
44.22\end{array}$ & $\begin{array}{l}39.8 \\
47.5 \\
40.0 \\
41.3 \\
40.0 \\
44.2 \\
38.5 \\
43.1\end{array}$ \\
\hline & \multicolumn{3}{|c|}{ Average } & & & 41.4 \\
\hline
\end{tabular}

* Calculated as "Standard" urea clearance but not included in average. $\uparrow$ Note different order in which diets were given. 
altered activity of the kidney in the excretion of urea. Increasing the protein intake above normal, however, did not result in a significant increase in clearance. In the one subject on whom protracted observations were made, the average urea clearance after 25 days on the high protein diet was slightly less than on the control diet.

Our observations on normal man confirm Cope's observations on nephritics with approximately normal urea clearances: the urea clearance is not significantly raised by a high protein diet, although it is significantly lowered by a low protein diet. Our observations show further that the lowering of the urea clearance is of the same order of magnitude, whether the clearances are determined above or below the augmentation limit. No explanation can be advanced for the fact that renal activity in man is so much less susceptible to dietary influence than is the case in the dog; it may be pointed out, however, that Jolliffe and Smith fed much larger quantities of protein (15 grams per kilogram of body weight) than our subjects consumed ( 4 grams per kilogram of body weight) and that in the experiments on the dog all the protein was supplied as meat whereas in man about onethird of the total protein intake is the most that can be conveniently ingested in this form.

\section{SUM MARY}

Observations on the urea clearance at urine flows above the augmentation limit in normal men subsisting on diets containing 9 or 280 grams of protein per day show a reduction in clearance at the lower protein level, but no change in clearance at the higher protein level as compared with a control period when the protein intake was 100 grams per day. These results are in agreement with Cope's observations on nephritics with approximately normal urea clearances, which observations were made, for the most part, when the urine flow was below the augmentation limit.

\section{BIBLIOGRAPHY}

1. Cope, C. L., Studies of urea excretion. VIII. The effects on the urea clearance of changes in protein and salt contents of the diet. J. Clin. Invest., $1933,-12,567$.

2. Jolliffe, N., and Smith, H. W., The excretion of urine in the dog. II. The urea and creatinine clearance on cracker meal diet. Am. J. Physiol., 1931, 99, 101.

3. Goldring, W., Studies of the kidney in acute infection. II. Observations with the urea clearance test in acute rheumatic fever. J. Clin. Invest., $1931,10,345$.

4. Van Slyke, D. D., Determination of urea by gasometric measurement of the carbon dioxide formed by the action of urease. J. Biol. Chem., 1927, 73, 695. 